\title{
Insights into the combination of neuromuscular electrical stimulation and motor imagery in a training-based approach
}

\author{
Amandine Bouguetoch $^{1}$ (1) Alain Martin ${ }^{1} \cdot$ Sidney Grosprêtre ${ }^{2}$
}

Received: 8 August 2020 / Accepted: 10 December 2020 / Published online: 8 January 2021

(c) The Author(s) 2021

\begin{abstract}
Introduction Training stimuli that partially activate the neuromuscular system, such as motor imagery (MI) or neuromuscular electrical stimulation (NMES), have been previously shown as efficient tools to induce strength gains. Here the efficacy of MI, NMES or NMES + MI trainings has been compared.

Methods Thirty-seven participants were enrolled in a training program of ten sessions in 2 weeks targeting plantar flexor muscles, distributed in four groups: MI, NMES, NMES + MI and control. Each group underwent forty contractions in each session, NMES + MI group doing 20 contractions of each modality. Before and after, the neuromuscular function was tested through the recording of maximal voluntary contraction (MVC), but also electrophysiological and mechanical responses associated with electrical nerve stimulation. Muscle architecture was assessed by ultrasonography.

Results MVC increased by $11.3 \pm 3.5 \%$ in NMES group, by $13.8 \pm 5.6 \%$ in MI, while unchanged for NMES + MI and control. During MVC, a significant increase in V-wave without associated changes in superimposed H-reflex has been observed for NMES and MI, suggesting that neural adaptations occurred at supraspinal level. Rest spinal excitability was increased in the MI group while decreased in the NMES group. No change in muscle architecture (pennation angle, fascicle length) has been found in any group but muscular peak twitch and soleus maximal M-wave increased in the NMES group only.

Conclusion Finally, MI and NMES seem to be efficient stimuli to improve strength, although both exhibited different and specific neural plasticity. On its side, NMES + MI combination did not provide the expected gains, suggesting that their effects are not simply cumulative, or even are competitive.
\end{abstract}

Keywords Strength $\cdot$ Triceps surae $\cdot$ Ultrasonography $\cdot$ Spinal excitability $\cdot$ V-wave

$\begin{array}{ll}\text { Abbreviations } \\ \text { ANOVA } & \text { Analysis of variance } \\ \mathrm{Ca}^{2+} & \text { Calcium ion } \\ \mathrm{CON} & \text { Control group } \\ \text { EME } & \text { Electromechanical efficiency } \\ \text { EMG } & \text { Electromyography } \\ \text { FL } & \text { Fascicle length } \\ \text { GL } & \text { Gastrocnemius lateralis } \\ \text { GM } & \text { Gastrocnemius medialis }\end{array}$

Communicated by Toshio Moritani.

Amandine Bouguetoch

amandine.bouguetoch@free.fr

Cognition, Action and Sensorimotor Plasticity [CAPS], Unité INSERM 1093, Université de Bourgogne-UFR STAPS, BP 27877, 21078 Dijon Cedex, France

2 EA-4660 C3S Culture Sport Health Society, University of Bourgogne Franche-Comté, Besançon, France

$\begin{array}{ll}H_{\mathrm{MAX}} & \text { Maximal H-reflex } \\ H_{\text {SUP }} & \text { Superimposed H-reflex } \\ M_{\text {ATH }} & \begin{array}{l}\text { M-wave recorded with the corresponding } \\ \text { H-reflex }\end{array} \\ \text { MI } & \text { Motor imagery } \\ \text { MIQ-R } & \text { Motor Imagery Questionnaire-Revised } \\ M_{\text {MAX }} & \text { Maximal M-wave } \\ M_{\text {SUP }} & \text { Superimposed M-wave } \\ \text { MT } & \text { Muscle thickness } \\ \text { NMES } & \text { Neuromuscular electrical stimulation } \\ \text { P } \alpha & \text { Pennation angle } \\ \text { Pt } & \text { Peak twitch } \\ \text { SOL } & \text { Soleus muscle } \\ \text { TA } & \text { Tibialis anterior } \\ \text { TTI } & \text { Torque time integral }\end{array}$




\section{Introduction}

It is now well established that training by resistance exercise in untrained individuals can induce muscle strength gains involving adaptations occurring within the muscle but also at the level of the neural drive (Aagaard 2003; Tallent et al. 2017; Kidgell et al. 2017). However, the significant mechanical muscle stress induced by heavy loads combined with a low number of repetitions does not seem to be the only strategy allowing strength gains in untrained subjects.

Indeed, there is now ample evidence demonstrating that motor imagery (MI), which consists in mentally simulating a movement without concomitant motor output (Jeannerod 1994), is also an effective way to improve motor performance and motor learning (Guillot and Collet 2008). This modality of training is efficient to induce force enhancements in upper and lower limb muscles (Paravlic et al. 2018). For instance, Zijdewind et al. (2003) but also Sidaway and Trzaska (2005) reported strength gains of more than $30 \%$ on the lower limbs after 4 and 7 weeks of MI training, respectively. Shorter training period with higher training frequency (daily routine) also showed significant force enhancements in the lower limb (Grosprêtre et al. 2017). However, MI solicits only a part of the neuromuscular system. Indeed, for a long time, strength gains following mental training have been attributed to a cortical reorganization only (Ranganathan et al. 2004). But more recently, it was shown that MI training may also induce plasticity at spinal level (Grosprêtre et al. 2017). The repetitive activation of cortical motor areas during MI, although at a subthreshold level since no motor output is observed, seems to impact spinal networks and more precisely the presynaptic neuronal circuitry (Grosprêtre et al. 2019). However, although a large portion of the brainto-muscle pathway seems to be involved, MI training do not provide motor output. This demonstrates that there is room for optimizing MI-training induced gains by combining it with other training modalities (e.g., neuromuscular electrical stimulation) leading to activation/recruitment of muscle fibers.

Neuromuscular electrical stimulation (NMES) is another alternative to physical training to improve muscle strength and is a widely used method in applied sports science or rehabilitation (Maffiuletti 2010). In contrast to a typical voluntary contraction initiated by the central nervous system (e.g., in resistance training), NMES consists in evoking contractions by applying an electrical current over the muscle via surface electrodes (Seyri and Maffiuletti 2011). With submaximal levels of evoked contraction, NMES strength gains were largely demonstrated in the literature, mostly on the lower limb (Laughman et al. 1983;
Gondin et al. 2005). Strength gains following NMES training (usually between 10 and 30\%, Hainaut and Duchateau 1992) have been obtained without significant changes in muscle mass or architecture, especially in the early phase of the training. This indicates that nervous mechanisms are involved in this improvement (Gondin et al. 2005, 2006) as evidenced by an enhancement of the voluntary muscle activation as measured with surface electromyography (Maffiuletti et al. 2002) or twitch interpolation method (Stevens et al. 2004). These neural effects may originate from a repetitive activation of afferent nervous circuitry, from the muscle up to the central nervous system. Despite some effects on the corticospinal network being observed with NMES, the incomplete activation of the motor system during NMES and the use of submaximal training intensities (Maffiuletti 2010) should partly account for its lower efficiency on muscle strength than resistance training (Laughman et al. 1983; Hainaut and Duchateau 1992). Thus, adding an efferent command without additional training loads (i.e. MI) could improve the gains and be applicable in rehabilitation purposes.

Therefore, both MI and NMES represent an incomplete activation of the neuromuscular system, but both sufficient to induce significant neural plasticity leading to improving muscle function. Saying that, the complementary gradients of MI and NMES neural activation led to the hypothesis that alternating both treatments in the same training session (NMES + MI) may provide additional effects compared to one of these modalities alone. The repetitive activation of cortical regions and corticomotoneuronal pathway provided by MI alternated with the repetitive afferent activation and motor output provided by NMES may lead to greater neural adaptations. The combination of both could therefore represent a promising training method. This alternating treatment could lead to an improvement of maximal strength without involving high training loads associated with voluntary resistance training. However, this approach has rarely been assessed and compared to NMES or MI alone. To date, little is known in the literature whether the effects provided by both methods can be complementary, particularly in a chronic approach. Training effects of such combination are not clear, because the different mechanisms involved in NMES and MI could also be antagonist and limit strength gains when applied in the same training session. Indeed, it has been previously shown that acute effects of MI (Grosprêtre et al. 2018) and NMES (Grosprêtre et al. 2019) can induce opposite modulation of presynaptic inhibition mechanisms.

The aim of this study was to assess strength gains following a training approach combining MI and NMES, and investigating the muscular and neural plasticity that results from this combination. This was compared to NMES and MI training performed alone with the same 
total number of repetitions and sessions. We can hypothesize that the summation of NMES and MI effects during training sessions would lead to greater enhancement of muscle strength, with a greater neural plasticity than these treatments applied alone, despite different neural mechanisms involved.

\section{Methods}

\section{Participants}

Thirty-seven healthy subjects (age: $24 \pm 5.8$ years old; height: $174 \pm 9 \mathrm{~cm}$; weight: $70 \pm 14 \mathrm{~kg}, 12$ females) gave written informed consent to participate in the present study and complied with the whole protocol. None of them reported any neurological or muscular disorders. There was no statistical difference between the groups of age, sex and physical activity pattern. Participants were recreationally active, and were asked not to perform any intense exercise during the training period. The experimental design was approved by the regional ethic committee (CPP COOM III number 2017-A00064-49; Clinical trial.gov identifier NCT03334526) and conducted in conformity with the latest version of the Declaration of Helsinki.

\section{General experimental design}

All participants performed a 2-week training on their right triceps surae preceded and followed by assessment of the neuromuscular function (Fig. 1). Participants were randomly distributed into 4 independent groups: control group (CON, $n=7,2$ females), neuromuscular electrical stimulation group (NMES, $n=10,3$ females), motor imagery group (MI, $n=10,4$ females) and combined group (NMES + MI, $n=10$, 3 females) according to the training they would undergo on their right plantar flexor muscles. While the control group did not perform any exercise during the duration of the protocol, each of the other groups followed 10 training sessions (5 per week) which is in the range of those previously found in the literature: Sidaway and Trzaska (2005) used 12 sessions of motor imagery, Grosprêtre et al. (2017) used 7 sessions of motor imagery, Gondin et al. (2006) used 15 sessions of NMES. For the trained groups, measurements were performed 3 days before the first training session and the day after the last training session, respectively for PRE- and POST-tests. Participants were familiarized to MI technique and/or to NMES during the inclusion visit at the laboratory, and during the first experimental session.

The same neurophysiological variables were measured in each of the four groups PRE- and POST-training (Fig. 1).

The PRE and POST tests consisted in performing maximal voluntary isometric contractions (MVC), ultrasound

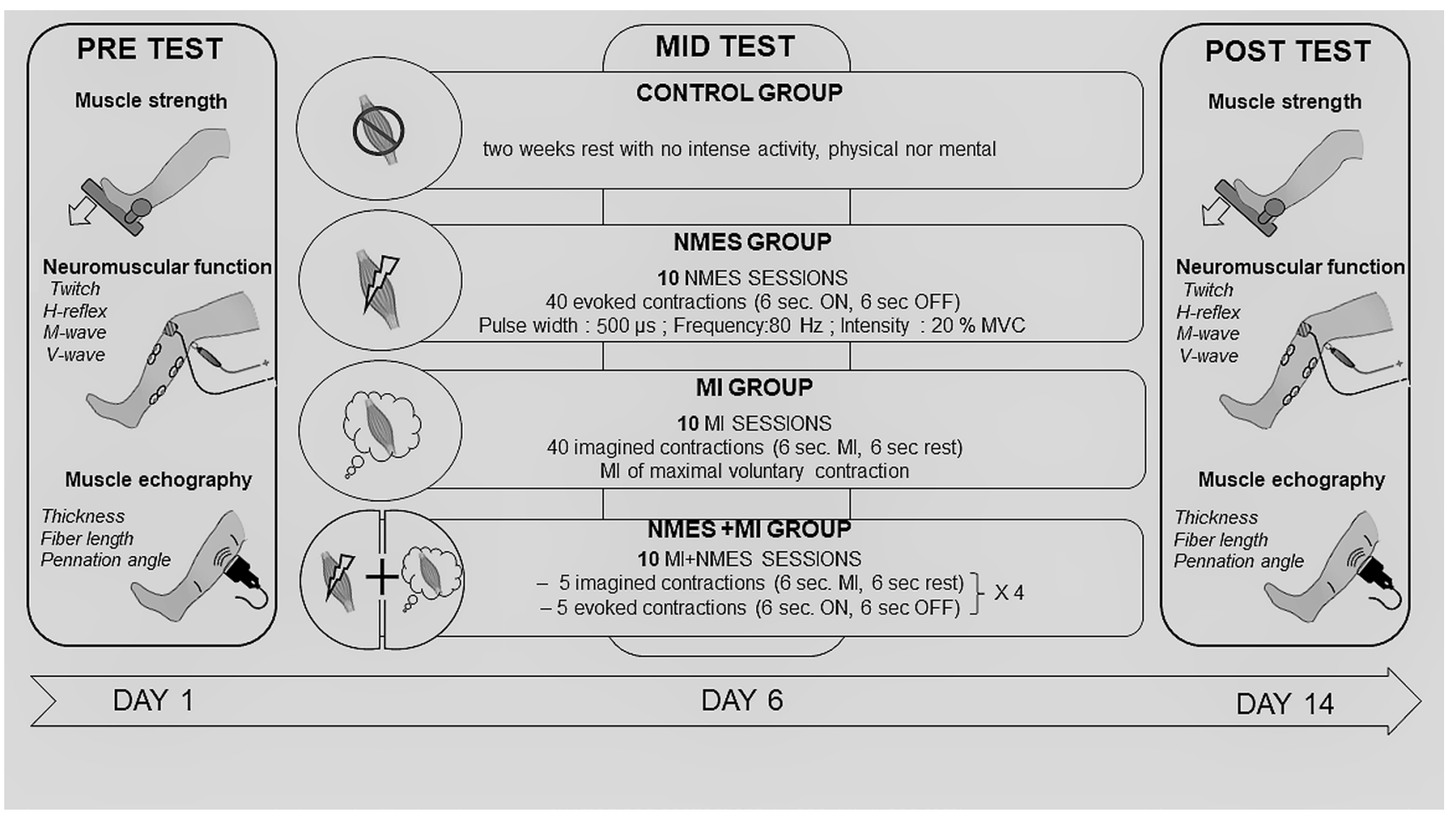

Fig. 1 Overview of the experimental protocol 
measurements and neuromuscular assessments. The testing sessions lasted $1 \mathrm{~h} 30$ in average and always took place in the laboratory between $9 \mathrm{~h} 30$ and $16 \mathrm{~h} 30$.

Each PRE and POST testing session started with muscle architecture assessment based on ultrasound measurements. During the rest of the testing session, participants seated on the chair of an isokinetic ergometer (Biodex system 4, Shirley, NY). The ergometer enabled instantaneous torque recording that was monitored throughout the experimental session. Electromyographic activities of the right triceps surae and tibialis anterior muscles were also recorded throughout the session.

Once installed, participants were asked to warm up their plantar flexor muscles by pushing sub-maximally against the pedal ten times with a force increment. Peripheral electrical nerve stimulations were sent to the posterior tibial nerve to elicit H-reflexes and M-waves of triceps surae.

\section{Training sessions}

Participants in NMES, MI and NMES + MI performed a 2-week training (10 days) consisting of isometric contractions on their right plantar flexor muscles. According to their group, the training was based on NMES, motor imagery or a combination of both. Participants in the control group did not train during this period. Each training session lasted 20-30 min and was conducted mainly between 12 and $14 \mathrm{~h}$ in the laboratory. During each training session, participants seated on the chair of the ergometer (Biodex system 4, Shirley, NY) with their right foot firmly tied on a pedal and set at a $90^{\circ}$-ankle angle as during PRE and POST tests.

\section{NMES training sessions}

Forty trains (6 s ON, 6 s OFF) of monophasic rectangular electrical stimuli were delivered to the triceps surae muscles by a high voltage $(400 \mathrm{~V})$ constant-current stimulator (Digitimer model DS7A, Hertfordshire, UK) triggered by a commercially available software (Tida, Heka Elektronik, Lambrecht/Pfalz, Germany). A pair of self-adhesive gel electrodes $(10 \times 5 \mathrm{~cm}$, Medicompex SA, Ecublens, Switzerland) was placed over the gastrocnemii $(\sim 5 \mathrm{~cm}$ below the popliteal fossa) and soleus $(\sim 10 \mathrm{~cm}$ above the calcaneus) muscles. Their contours were traced with a permanent marker to ensure that electrodes were always placed in the same position. Each train consisted in $6 \mathrm{~s}$ of electrical pulses with duration fixed at $500 \mu \mathrm{s}$, evoked at a constant frequency of $80 \mathrm{~Hz}$. Before each training session, pulse intensity was set to evoke an initial force level corresponding to $20 \%$ of the MVC (10-45 mA) using 3 testing trains of $2 \mathrm{~s}$, adapted to each participant. This intensity was not modified during the session.

\section{MI training sessions}

Each participant who practiced MI first completed the revised version of the Movement Imagery Questionnaire (MIQ-R Hall and Martin 1997) to determine selfestimation of MI ability. The initial mean MIQ-R score was $46.1 \pm 6.1$ (maximum score: 56 ), indicating a good imagery capacity in all participants. Participants performed each MI training session in the laboratory under the supervision of the same experimenter. MI training sessions consisted of 40 MI of maximal isometric plantar flexion of the right leg during $6 \mathrm{~s}$, interspaced with a rest period of $6 \mathrm{~s}$. Participants were instructed to imagine pushing maximally on the pedal of the ergometer, and to associate the mental representation of the sensations normally experienced during actual performance (kinesthetic modality). Each imagined trial was preceded and stopped by an auditory signal. The continuous recording of force signal and background electromyography (EMG) of triceps surae muscles helped to ensure that no motor output was provided during the completion of the MI task and that participants stayed fully relaxed. Participants were asked to rate the quality of their MI training session with a quote from 1 (poor) to 10 (excellent). During the training, the self-estimated imagery (mean: $7 \pm 2$ out of 10 ) did not vary significantly.

\section{Combined MI + NMES training sessions}

The NMES + MI group underwent 20 MI and 20 evoked contractions for a total of 40 contractions like the other training programs. These contractions were implemented in 4 blocks of 5 evoked contractions ( $6 \mathrm{~s}$ ON and $6 \mathrm{~s}$ OFF) and 5 imagined contractions (6 s MI and $6 \mathrm{~s}$ rest) in this order. Each MI and NMES trials were performed with the same modality of single NMES and MI training described above. The initial mean MIQ-R score was $44.1 \pm 5.4$ (maximum score: 56). During training, self-estimated imagery (mean: $7 \pm 2$ out of 10 ) did not vary significantly and the stimulation intensity range was $10-45 \mathrm{~mA}$, adapted to each participant and reassessed at the beginning of each training session to match $20 \%$ MVC.

In both NMES and NMES + MI groups, MVC was reassessed during the first and the sixth sessions to readjust NMES intensity to still correspond to the same percentage of the actualized MVC performance. To standardize the protocol, MI and CONTROL groups also performed 2 MVC during the first and the sixth sessions. Before these two training sessions, participants warmed-up by pushing sub-maximally 10 times against the pedal, then performed 2 maximal isometric contractions in the same conditions as PRE and POST tests. 


\section{Mechanical recordings}

The torque of the participant during each actual and evoked contraction was measured by means of an isokinetic dynamometer (Biodex system 4, Shirley, NY). The axis of the dynamometer was aligned with the right external malleolus. Subjects were placed with the hip and knee joints at $90^{\circ}\left(180^{\circ}=\right.$ full extension), and ankle joint at $90^{\circ}$ (angle between the leg and the sole). During all conditions, care was taken to avoid trunk and head rotations to maintain constant corticovestibular influences on the excitability of the motor pool (Schieppati 1987). The foot was firmly strapped to the pedal of the dynamometer in two places, i.e. the end of the metatarsus and the junction with the shinbone, to prevent the heel from lifting. The trunk was stabilized by two crossover shoulder harnesses. Torque signal was recorded continuously during the whole protocol.

\section{Electromyography (EMG) recording}

EMG activity was recorded from four muscles of the leg (soleus: SOL, gastrocnemius medialis: GM, gastrocnemius lateralis: GL; tibialis anterior: TA). After shaving and dry-cleaning the skin with alcohol to keep a low impedance $(<5 \mathrm{k} \Omega)$, EMG signals were recorded using two silver chloride surface electrodes ( $8 \mathrm{~mm}$ diameter) placed at an interelectrode center-to-center distance of $2 \mathrm{~cm}$. For SOL, electrodes were placed $2 \mathrm{~cm}$ below the insertions of the gastrocnemii over the Achille's tendon; for GM and GL, over the mid belly of the muscles; and for TA, at $1 / 3$ of the distance between the fibula and the lateral malleolus. A common reference electrode was placed between stimulation and recording sites on the lower part of the belly of the gastrocnemii. EMG signals were amplified with a bandwidth frequency ranging from $15 \mathrm{~Hz}$ to $1 \mathrm{kHz}$ (gain=1000) then digitized online (sampling frequency: $5 \mathrm{kHz}$ ) using the MP150 Biopac system and stored for analysis with Acqknowledge software 4.2.

\section{Muscle architecture}

Participants lay in a static prone position for $10 \mathrm{~min}$ before any measure was taken. Then, a 5.5-cm $(7.5 \mathrm{MHz})$ linear array probe was positioned perpendicular to the dermal surface and oriented along the longitudinal axis of the muscle-tendon unit. The GL upper insertion was found in the popliteal fossa and marked. A measure was taken from this mark to reach the belly of the GL and transferred to the GM as well. This distance $(10-14 \mathrm{~cm})$ was noted on the skin with a permanent marker for POST tests. Three images of each muscle were recorded using B-mode
Zonare ultrasound video imaging (Z. One ultra sp 4.2, Zonare Medical Systems Inc. Mountain View, CA, USA). Fascicle length (FL), pennation angle ( $\mathrm{P} \alpha)$ and muscle thickness (MT) were extracted. Criteria for storing images were parallel superficial and deep aponeurosis, and the presence of at least three discernible fascicles with their junction upon deep aponeurosis. On each image, MT was measured from the average distance between the deep and the superficial aponeurosis measured directly by the ultrasound software. P $\alpha$ and FL were calculated.

\section{Neuromuscular assessment}

Right posterior tibial nerve percutaneous nerve stimulations were used to record $\mathrm{H}$-reflexes and $\mathrm{M}$-waves of the considered muscles. Single rectangular pulses (1-ms width) were delivered by a high voltage $(400 \mathrm{~V})$ constantcurrent stimulator, through a self-adhesive anode $(8-\mathrm{mm}$ diameter, $\mathrm{Ag}-\mathrm{AgCL}$ ) placed in the popliteal fossa and a cathode $(5 \times 10 \mathrm{~cm}$, Medicompex SA, Ecublens, Switzerland) placed over the patella. Optimal stimulation site was first located by a hand-held ball electrode $(0.5-\mathrm{cm}$ diameter) to obtain the greatest H-reflex amplitudes for the lowest stimulation intensity, focusing on the SOL muscle. Particular care was taken in avoiding EMG responses on the antagonist muscle (TA). Once determined, stimulation electrodes were firmly fixed to the optimal site with straps.

To build $\mathrm{H}-\mathrm{M}$ recruitment curves, stimulations started at the H-reflex threshold and were progressively increased with a $4 \mathrm{~mA}$ increment until $\mathrm{M}$-wave amplitude no longer increased. To ensure that the M-wave lay in the plateau of its maximal value, maximal stimulation intensity was increased by $20 \%$ and taken as $M_{\mathrm{MAX}}$ intensity. Three responses were evoked at each intensity, with an interstimulus interval of $10 \mathrm{~s}$. Any response with a voluntary pre-stimulus was removed. The whole curve from H-reflex threshold to $M_{\mathrm{MAX}}$ was performed PRE and POST training.

The intensity of the maximal H-reflex, noted $H_{\mathrm{MAX}}$ at rest and $H_{\text {SUP }}$ during contraction, was used to assess spinal excitability during maximal contractions (MVC). As well, superimposed twitches at $M_{\mathrm{MAX}}$ intensity were evoked during MVC of the plantar flexor muscles. Contrary to rest, it can be noticed that the evoked maximal M-wave $\left(M_{\text {SUP }}\right)$ is accompanied by a reflex wave noted $\mathrm{V}$-wave, which is an electrophysiological variant of the H-reflex virgules noter. Stimulation were manually triggered once the torque lay in the plateau of its maximal value. Participants were asked to maintain the contraction for $1 \mathrm{~s}$ after the stimulation. Two stimulations were performed for $H_{\text {SUP }}$ and two for $M_{\mathrm{Sup}} / \mathrm{V}$-wave during separated MVC, with at least 1-min rest between. 


\section{Data analysis}

\section{Mechanical responses}

The maximal force was measured as the peak of the torque developed during MVCs when the signal lay in the plateau of its maximal value. Within subjects data were carefully checked for extremes values i.e., different from more than two standard deviations of the mean. Therefore, over the six to eight trials performed for MVC measurements at each time point (PRE and POST), 2 values of 1 participant in the MI group and 2 values of 2 participants in the NMES group were discarded. These discarded trials, showing abnormally high MVC values, were attributed to a high recruitment of the quadriceps and/or the gluteal muscles during the completion of the plantar flexion, therefore compensating with the triceps surae muscle recruitment. It should be noticed that this correction has been performed for MVC only, other data not showing such extreme values. Delta values of strength gains were calculated as (POST-values - PRE-values)/PRE-values $\times 100$.

Mechanical twitches following maximal M-wave stimulations at rest were also analyzed to account for peripheral changes. Twitches were measured as the peak amplitude of the torque signal. The electromechanical efficiency (EME) was then calculated by the ratio of this peak twitch (Pt) over the sum of amplitudes of the three corresponding maximal M-waves, i.e., SOL, GM and GL (Pt/ $\left.M_{\mathrm{MAX}}\right)$. For NMES and NMES + MI, the area under the curve of each electrically evoked contraction, i.e., the torque-time integral (TTI), was determined during all training sessions. The TTI of all evoked contractions was summed for each training session in NMES and NMES + MI groups to express the total TTI.

\section{Electrophysiological responses}

Peak-to-peak amplitudes of each EMG response at rest and superimposed to MVC (M-waves, H-reflexes, V-waves) were measured and averaged among the different trials. The amplitude of the submaximal M-wave accompanying each H-reflex, noted $M_{\mathrm{atH}}$, was also considered.

Rest recruitment curves of $\mathrm{H}$ - and $\mathrm{M}$-waves were built for each muscle of the triceps surae by plotting stimulus intensities against response amplitudes. All H-reflexes and submaximal $\mathrm{M}$-wave values were normalized by the corresponding maximal M-wave. As well, the intensity necessary to obtain each response was expressed as a percentage of the maximal intensity (i.e., $M_{\mathrm{MAX}}$ intensity). Mean recruitment curves over all participants were built by sorting out the $H-M$ values by ranges of $10 \%$ of $M_{\mathrm{MAX}}$ intensity. The average of all the participants in each range was used for statistical analysis performed on the recruitment curves.

RMS was measured during MVCs and normalized by maximal M-wave amplitude measured during $\mathrm{MVC}\left(M_{\mathrm{SUP}}\right)$.

\section{Muscle architecture}

For each subject, three images of GM and GL muscles were recorded. On each of them, we measured three architectural parameters with the ultrasound software. The first one was muscle thickness taken between the deep and the superficial aponeurosis, perpendicularly to the deep aponeurosis, on the left and right edges, and at the middle of the image. The second measure called d1 was the partial fascicle length. Indeed, for most of the subjects, muscle fascicles could not be directly measured by the software since fascicles were longer than the screen of the ultrasound device. The third measure called $\mathrm{d} 2$ was the distance between the deep aponeurosis and the end of the partial fascicle, taken perpendicularly to the deep aponeurosis. Thus, $\mathrm{d} 1$ and $\mathrm{d} 2$ designed a squared triangle with the deep aponeurosis, $\mathrm{d} 1$ being the hypotenuse. From these measurements, pennation angle and fascicle length were obtained by calculating $P \alpha=\arcsin (\mathrm{d} 2 /$ d1) and $F L=$ muscle thickness/sin $(\mathrm{P} \alpha)$, respectively. At last, three measurements from the three images were averaged for each subject and each muscle.

\section{Statistical analysis}

All data are presented as the mean \pm standard deviation. Shapiro-Wilk test $(p<0.05)$ was used to ensure the normality and homogeneity was assessed by means of Levene test. Each muscle was assessed separately. A two-way ANOVA with repeated measures was performed on each dependent variable with the factor group (CONTROL, MI, NMES, and $\mathrm{NMES}+\mathrm{MI}$ ) and the factor time (PRE and POST). When a main or interaction effect was found, a post-hoc analysis was conducted using Tukey's HSD (honest significant difference) test. Statistical analysis was performed with Statistica software (10.0 version, Statsoft, Tulsa, Oklahoma, USA). The level of significance was set at $p<0.05$.

\section{Results}

\section{Strength}

A significant time $\times$ group interaction was found for the maximal torque $\left(F_{3,33}=4.129, p=0.013\right)$. Pre-values were not statistically different between groups (all $p>0.53$ ). The maximal torque was significantly increased by $11.3 \pm 3.5 \%$ for the NMES group and by $13.8 \pm 5.6 \%$ for the MI group 


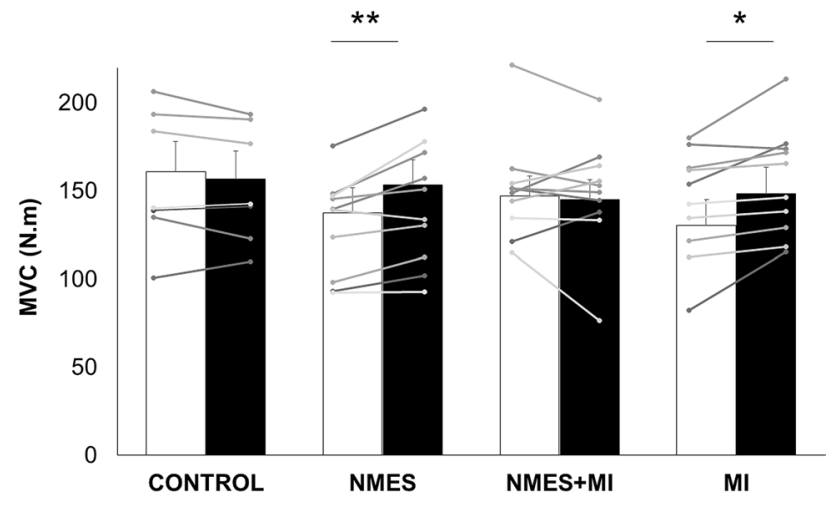

Fig. 2 Plantar flexor muscles force characteristics of the four groups. Data are depicted as mean \pm SD for the four groups: the control group (CONTROL) and the three trained groups: neuromuscular electrical stimulation only (NMES), combined NMES and motor imagery (NMES + MI) and MI only (MI). Maximal torque of the plantar flexor muscles recorded during maximal voluntary contraction (MVC) before (PRE, white bars) and after (POST, black bars) the training protocols. Individual data are depicted in gray dots and lines. ${ }^{*}$,*Significant PRE-POST difference at $p<0.05$ and $p<0.01$, respectively

(Fig. 2a). A statistical analysis of these PRE-POST deltas revealed a significant effect of the factor group $\left(F_{3,33}=12.42\right.$, $p<0.001)$. PRE-to-POST changes were significantly higher in NMES and MI groups as compared to CONTROL ( $p<0.001$ and $p=0.0023$, respectively) and NMES + MI ( $p=0.004$ and $p=0.002$, respectively). There was no difference between NMES and MI $(p=0.934)$.

For the NMES group, the total TTI among the ten training sessions was $20423.2 \pm 6794.8 \mathrm{Nm}$ s. The averaged total TTI for the NMES + MI group was significantly lower (17 441.6 8 990.4 Nm s, $p=0.003$ ).

\section{Muscular parameters}

No main effect or interaction was found for any of the architectural parameters measured by ultrasound, such as MT, P $\alpha$ or FL (Table 1). No parameter was modified after any of the tested training or for the control group.

On the contrary, a significant time $\times$ group interaction was found for the plantar flexor muscles peak twitch associated with supramaximal stimulation $\left(F_{3,33}=3.82\right.$, $p=0.019)$. The peak twitch was increased from PRE to POST only after NMES training $(p=0.011)$, from $21.9 \pm 7.9$ to $26.5 \pm 8.9 \mathrm{~N} \mathrm{~m}$, and remained unchanged for the three other groups (CONTROL: from 24.5 \pm 7.2 to $25.5 \pm 8.5 \mathrm{~N} \mathrm{~m}$; MI: from $20.8 \pm 8.9$ to $22.4 \pm 6.3 \mathrm{~N} \mathrm{~m}$; NMES + MI: from $21.6 \pm 7.7$ to $21.2 \pm 6.6 \mathrm{~N} \mathrm{~m}$ ). A significant time $\times$ group interaction was found for maximal muscle action potential, i.e. $M_{\mathrm{MAX}}$ amplitude, for SOL muscle only $\left(F_{3,33}=4.353, p=0.011\right)$. SOL maximal M-wave was increased after NMES training only $(p=0.018)$, from $12.3 \pm 3.7$ to $14.8 \pm 3.7 \mathrm{mV}$ (Table 2 ), while no change has been found in the three other groups. As well, a significant increase in EME has been found for NMES group only ( $p=0.009$ ), from $0.76 \pm 0.27$ to $0.89 \pm 0.29 \mathrm{~N} \mathrm{~m} / \mathrm{mV}$ (CONTROL: from $0.82 \pm 0.2$ to $0.81 \pm 0.2 \mathrm{~N} \mathrm{~m} / \mathrm{mV}$; $\mathrm{MI}$ : from $0.70 \pm 0.3$ to $0.73 \pm 0.3 \mathrm{~N} \mathrm{~m} / \mathrm{mV}$; $\mathrm{NMES}+\mathrm{MI}$ : from $0.82 \pm 0.3$ to $0.83 \pm 0.3 \mathrm{~N} \mathrm{~m} / \mathrm{mV})$.

\section{Nervous parameters}

Regarding the recruitment curves, while no change was observed either in the control or in the NMES + MI group, some PRE-POST differences have been found in the two other groups (Fig. 3).

In the NMES group, a significant decrease in $H / M_{\text {MAX }}$ ratios including $H_{\mathrm{MAX}} / M_{\mathrm{MAX}}$ has been observed in the three muscles from PRE to POST at $40 \%$ (SOL: $p=0.025$; GM: $p=0.016$; GL: $p=0.047)$ and $50 \%$ of maximal intensity (SOL: $p=0.020$; GM: $p=0.028$; GL: $p=0.045$ ). The associated $M_{\mathrm{atH}} / M_{\mathrm{MAX}}$ were significantly higher in POST than in PRE for those two intensities (40\%: SOL: $p=0.022$; GM: $\mathrm{p}=0.036$; GL: $p=0.044 ; 50 \%$ : SOL: $p=0.016$; GM: $p=0.035$; GL: $p=0.022$ ). This PRE-to-POST decrease of
Table 1 Ultrasound data including muscle thickness $(\mathrm{cm})$, pennation angle $\left(^{\circ}\right)$ and fascicle length $(\mathrm{cm})$

\begin{tabular}{|c|c|c|c|c|c|c|}
\hline & \multicolumn{2}{|c|}{ Thickness } & \multicolumn{2}{|c|}{ Pennation angle } & \multicolumn{2}{|l|}{ Fiber length } \\
\hline & PRE & POST & PRE & POST & PRE & POST \\
\hline \multicolumn{7}{|c|}{ Gastrocnemius medialis } \\
\hline MI & $1.8 \pm 0.3$ & $1.8 \pm 0.3$ & $19.9 \pm 2.3$ & $21.2 \pm 6.4$ & $5.5 \pm 0.8$ & $5.3 \pm 1.1$ \\
\hline NMES & $2.0 \pm 0.3$ & $2.0 \pm 0.2$ & $19.5 \pm 2.7$ & $20.8 \pm 2.6$ & $5.9 \pm 0.7$ & $5.6 \pm 0.6$ \\
\hline NMES + MI & $1.8 \pm 0.2$ & $1.7 \pm 0.3$ & $20.9 \pm 3.7$ & $19.2 \pm 3.5$ & $5.3 \pm 0.8$ & $5.3 \pm 0.9$ \\
\hline CONTROL & $2.1 \pm 0.2$ & $2.1 \pm 0.3$ & $23.0 \pm 3.8$ & $20.9 \pm 3.4$ & $5.6 \pm 1.1$ & $6.0 \pm 1.1$ \\
\hline \multicolumn{7}{|c|}{ Gastronemius lateralis } \\
\hline MI & $1.4 \pm 0.3$ & $1.4 \pm 0.2$ & $13.3 \pm 2.0$ & $14.7 \pm 2.4$ & $6.1 \pm 1.5$ & $5.7 \pm 0.7$ \\
\hline NMES & $1.5 \pm 0.3$ & $1.5 \pm 0.3$ & $14.4 \pm 3.2$ & $13.9 \pm 2.1$ & $6.0 \pm 0.9$ & $6.3 \pm 0.5$ \\
\hline NMES + MI & $1.4 \pm 0.4$ & $1.3 \pm 0.3$ & $13.2 \pm 4.3$ & $12.0 \pm 2.9$ & $6.3 \pm 1.6$ & $6.2 \pm 1.7$ \\
\hline CONTROL & $1.5 \pm 0.2$ & $1.4 \pm 0.2$ & $12.7 \pm 2.9$ & $11.5 \pm 2.7$ & $7.1 \pm 2.0$ & $7.2 \pm 1.8$ \\
\hline
\end{tabular}


Table 2 Mean \pm SD raw values for the four groups $(\mathrm{mV})$

\begin{tabular}{|c|c|c|c|c|c|c|c|c|}
\hline & \multicolumn{2}{|l|}{ CONTROL } & \multicolumn{2}{|l|}{ NMES } & \multicolumn{2}{|c|}{$\mathrm{NMES}+\mathrm{MI}$} & \multicolumn{2}{|l|}{ MI } \\
\hline & PRE & POST & PRE & POST & PRE & POST & PRE & POST \\
\hline \multicolumn{9}{|l|}{ SOL } \\
\hline$H_{\text {MAX }}$ & $4.4 \pm 1.6$ & $4.5 \pm 2.0$ & $4.7 \pm 2.1$ & $3.3 \pm 2.1$ & $4.0 \pm 2.5$ & $4.7 \pm 2.2$ & $3.4 \pm 1.5$ & $4.6 \pm 2.6$ \\
\hline$M_{\text {atHmax }}$ & $2.2 \pm 1.1$ & $2.0 \pm 1.1$ & $2.1 \pm 1.2$ & $4.0 \pm 0.8$ & $2.1 \pm 1.6$ & $2.1 \pm 1.4$ & $2.1 \pm 1.5$ & $2.1 \pm 1.4$ \\
\hline$M_{\mathrm{MAX}}$ & $9.8 \pm 4.0$ & $10.4 \pm 1.8$ & $12.3 \pm 3.7$ & $14.8 \pm 3.7$ & $11.4 \pm 4.5$ & $11.1 \pm 4.4$ & $10.6 \pm 4.7$ & $10.9 \pm 3.4$ \\
\hline$H_{\text {SUP }}$ & $5.9 \pm 1.4$ & $6.3 \pm 1.1$ & $6.7 \pm 3.3$ & $6.0 \pm 1.7$ & $5.7 \pm 2.9$ & $5.2 \pm 3.6$ & $7.1 \pm 3.7$ & $6.9 \pm 5.1$ \\
\hline$M_{\text {atHsup }}$ & $2.1 \pm 2.3$ & $1.6 \pm 0.9$ & $3.7 \pm 2.5$ & $3.0 \pm 2.4$ & $1.2 \pm 1.0$ & $1.8 \pm 1.7$ & $3.8 \pm 2.4$ & $3.2 \pm 2.9$ \\
\hline$M_{\mathrm{SUP}}$ & $12.1 \pm 3.3$ & $12.7 \pm 2.2$ & $12.0 \pm 3.7$ & $12.8 \pm 3.7$ & $13.0 \pm 4.0$ & $12.9 \pm 3.0$ & $13.3 \pm 3.0$ & $13.5 \pm 2.9$ \\
\hline$V$ & $4.6 \pm 1.0$ & $3.9 \pm 0.7$ & $3.6 \pm 1.9$ & $5.7 \pm 2.0$ & $3.4 \pm 1.8$ & $4.6 \pm 2.9$ & $3.0 \pm 2.9$ & $6.5 \pm 4.1$ \\
\hline \multicolumn{9}{|l|}{ GM } \\
\hline$H_{\mathrm{MAX}}$ & $1.5 \pm 0.7$ & $1.9 \pm 1.0$ & $1.7 \pm 1.2$ & $1.2 \pm 1.0$ & $1.1 \pm 0.8$ & $1.2 \pm 0.7$ & $1.3 \pm 1.1$ & $2.1 \pm 0.8$ \\
\hline$M_{\text {atHmax }}$ & $3.4 \pm 1.5$ & $3.3 \pm 2.1$ & $4.3 \pm 2.4$ & $6.4 \pm 3.0$ & $3.5 \pm 1.1$ & $3.6 \pm 2.5$ & $3.1 \pm 2.0$ & $3.1 \pm 2.3$ \\
\hline$M_{\mathrm{MAX}}$ & $5.7 \pm 2.0$ & $5.9 \pm 2.1$ & $6.8 \pm 1.9$ & $7.3 \pm 2.8$ & $6.1 \pm 4.4$ & $5.3 \pm 2.0$ & $7.1 \pm 4.7$ & $6.7 \pm 3.4$ \\
\hline$H_{\text {SUP }}$ & $3.1 \pm 1.1$ & $3.2 \pm 1.6$ & $3.5 \pm 1.9$ & $3.6 \pm 1.6$ & $2.4 \pm 1.2$ & $2.1 \pm 1.1$ & $3.4 \pm 2.3$ & $3.2 \pm 1.8$ \\
\hline$M_{\text {atHsup }}$ & $2.3 \pm 1.7$ & $3.2 \pm 2.2$ & $3.60 \pm 3.0$ & $4.0 \pm 4.1$ & $1.7 \pm 1.3$ & $2.6 \pm 1.7$ & $4.9 \pm 3.8$ & $4.1 \pm 3.2$ \\
\hline$M_{\text {SUP }}$ & $8.8 \pm 3.0$ & $8.2 \pm 2.9$ & $10.3 \pm 4.6$ & $10.8 \pm 4.1$ & $7.3 \pm 4.5$ & $6.4 \pm 3.7$ & $9.8 \pm 4.5$ & $9.5 \pm 3.3$ \\
\hline V & $2.1 \pm 1.1$ & $1.8 \pm 1.1$ & $1.8 \pm 1.4$ & $2.7 \pm 1.2$ & $2.1 \pm 0.5$ & $2.4 \pm 0.6$ & $2.0 \pm 2.3$ & $3.2 \pm 1.6$ \\
\hline \multicolumn{9}{|l|}{ GL } \\
\hline$H_{\text {MAX }}$ & $1.3 \pm 0.5$ & $1.6 \pm 0.8$ & $2.0 \pm 2.1$ & $1.0 \pm 1.0$ & $1.3 \pm 0.9$ & $1.3 \pm 0.9$ & $1.2 \pm 0.8$ & $1.6 \pm 0.8$ \\
\hline$M_{\text {atHmax }}$ & $3.2 \pm 2.7$ & $4.7 \pm 2.9$ & $4.0 \pm 3.9$ & $5.3 \pm 3.4$ & $4.3 \pm 4.4$ & $4.1 \pm 3.3$ & $5.6 \pm 3.5$ & $5.6 \pm 4.3$ \\
\hline$M_{\mathrm{MAX}}$ & $8.0 \pm 1.6$ & $8.4 \pm 0.7$ & $10.8 \pm 4.0$ & $8.8 \pm 2.6$ & $10.3 \pm 5.4$ & $9.0 \pm 4.1$ & $8.8 \pm 3.2$ & $9.2 \pm 3.1$ \\
\hline$H_{\text {SUP }}$ & $2.8 \pm 0.8$ & $2.8 \pm 1.2$ & $3.5 \pm 2.7$ & $3.0 \pm 1.7$ & $3.2 \pm 2.8$ & $2.7 \pm 2.4$ & $2.1 \pm 1.3$ & $2.5 \pm 1.5$ \\
\hline$M_{\text {atHsup }}$ & $2.6 \pm 3.0$ & $2.9 \pm 2.8$ & $3.6 \pm 3.1$ & $3.0 \pm 1.2$ & $2.9 \pm 2.0$ & $3.3 \pm 2.9$ & $3.1 \pm 2.0$ & $3.9 \pm 2.9$ \\
\hline$M_{\text {SUP }}$ & $12.1 \pm 3.3$ & $10.5 \pm 2.6$ & $12.7 \pm 4.2$ & $10.1 \pm 3.2$ & $13.0 \pm 4.0$ & $11.5 \pm 3.3$ & $12.7 \pm 3.7$ & $11.0 \pm 3.9$ \\
\hline$V$ & $1.7 \pm 0.7$ & $1.8 \pm 1.0$ & $1.9 \pm 1.5$ & $3.1 \pm 1.3$ & $1.9 \pm 1.5$ & $1.5 \pm 0.7$ & $2.0 \pm 1.8$ & $3.0 \pm 1.3$ \\
\hline
\end{tabular}

$H / M_{\mathrm{MAX}}$ ratios at 40 and $50 \%$ of maximal intensity was significantly correlated to the increase of $M_{\mathrm{atH}} / M_{\mathrm{MAX}}$ ratios at the same ranges of intensities (SOL: $r=0.96$; GM: $r=0.87$; GL: $r=0.71$ ).

In the MI group, a significant PRE-to-POST increase in $H / M_{\mathrm{MAX}}$ ratios including $H_{\mathrm{MAX}} / M_{\mathrm{MAX}}$ was observed at $30 \%$ and $40 \%$ of maximal intensity in SOL $(30 \%: p=0.041$ and 40\%: $p=0.023)$ and in GM (30\%: $p=0.033$ and 40\%: $p=0.018)$, with no change in $M_{\mathrm{atH}} / M_{\mathrm{MAX}}$.

A significant time $\times$ group interaction has been found for $V / M_{\text {SUP }}$ in SOL $\left(F_{3,33}=4.465, p=0.010\right)$, in $\mathrm{GM}\left(F_{3,33}=3.562, p=0.024\right)$ and in $\operatorname{GL}\left(F_{3,33}=5.114\right.$, $p=0.005)$. As depicted in Fig. $4 \mathrm{~V} / \mathrm{M}_{\mathrm{SUP}}$ ratios were significantly enhanced after NMES training (SOL: $p=0.012$; GM: $p=0.474$; GL: $p=0.007$ ) and after MI training (SOL: $p=0.004$; GM: $p=0.002$; GL: $p=0.033$ ).

No significant main effect or interaction was found for SOL superimposed H-reflex, i.e. $H_{\mathrm{SUP}} / M_{\mathrm{SUP}}\left(F_{3,33}=1.129\right.$, $p=0.352)$, neither for $\mathrm{GM}\left(F_{3,33}=1.066, p=0.377\right)$ nor $\mathrm{GL}$ $\left(F_{3,33}=0.226, p=0.878\right)$.

A significant interaction was found on muscular activity recorded during $\mathrm{MVC}\left(\mathrm{RMS} / M_{\mathrm{SUP}}\right)$, for $\mathrm{SOL}$ $\left(F_{3,33}=5.175, p=0.005\right), \mathrm{GM}\left(F_{3,33}=5.943, p=0.002\right)$ and $\operatorname{GL}\left(F_{3,33}=3.291, p=0.033\right)$. RMS $/ M_{\mathrm{SUP}}$ was increased after NMES and MI training for SOL $(p=0.003$ and $p=0.020$, respectively), GM ( $p=0.010$ and $p=0.013$ ) and GL $(p=0.035$ and $p=0.019)$.

\section{Discussion}

The present study investigated the effect of a 2-week intervention program on plantar flexor muscles with alternated NMES + MI compared to MI and NMES alone. The maximal performance, i.e. MVC, only increased for MI and NMES groups while no change has been found in the combined NMES + MI group. This was accompanied with supraspinal changes, as evidenced by an increased $V / M_{\text {SUP }}$ in SOL, GM and GL for both NMES and MI groups without any modifications of $H_{\mathrm{SUP}} / M_{\mathrm{SUP}}$. Spinal plasticity at rest occurred for both MI and NMES groups but in different directions. While a decrease in $H / M_{\text {MAX }}$ was observed in the NMES group, an increase in $H / M_{\mathrm{MAX}}$ was found in SOL and GM in the MI group. No change in muscle architecture has been found in any group. The combined group did not exhibit either neural or muscular changes. 


\section{$-\bigcirc-P R E$}

- POST

CONTROL

NMES
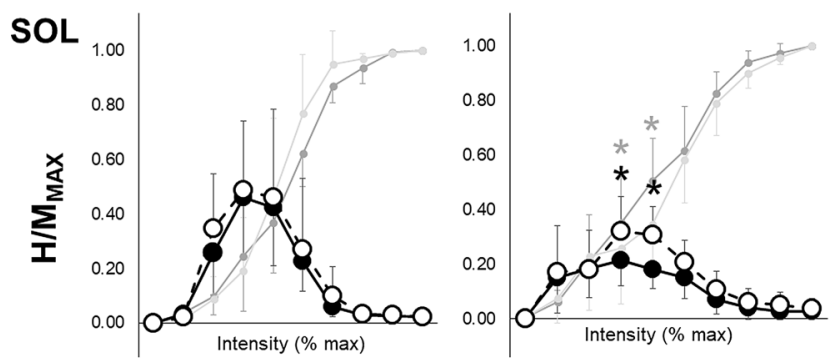

GM
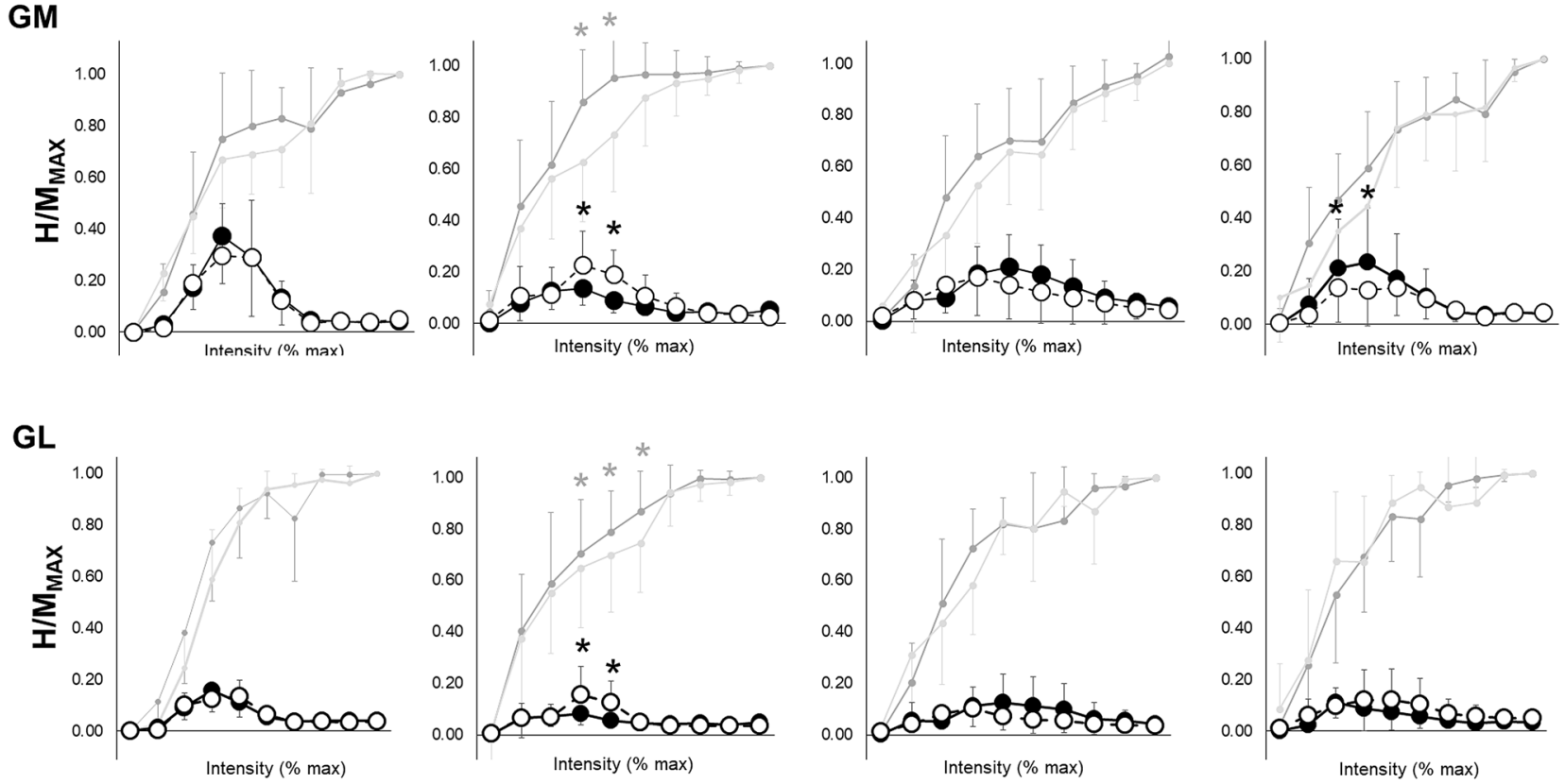

Fig. 3 Spinal excitability of the plantar flexor muscles in the four groups. Spinal excitability is expressed as the recruitment curves the $\mathrm{H}$-reflexes and $\mathrm{M}$-waves of the soleus (SOL), gastrocnemius medialis $(\mathrm{GM})$ and gastrocnemius lateralis (GL) recorded for the four groups (CONTROL; NMES neuromuscular electrical stimulation only, MI motor imagery, $N M E S+M I$ combined training). Recruitment curves are built as follows: mean H-reflex responses are normalized by the corresponding maximal $\mathrm{M}$-wave $\left(M_{\mathrm{MAX}}\right)$ in ten portions of intensities

\section{NMES training alone}

In the present study, significant strength gains were observed in the NMES group with an increase in MVC by $11.3 \pm 3.5 \%$ after 2 weeks of training at a targeted force of $20 \%$ MVC. Changes at central level were observed, whereas NMES training did not involve changes in muscle architecture.

Strength gains following NMES training are widely reported in the literature. Despite different methodologies in terms of stimulation site, targeted evoked force, pulse width and frequencies, strength gains vary from 8 to $22 \%$ for plantar flexors (Maffiuletti et al. 2001; Gondin et al. 2006). Similar strength gains have been reported by Gondin and
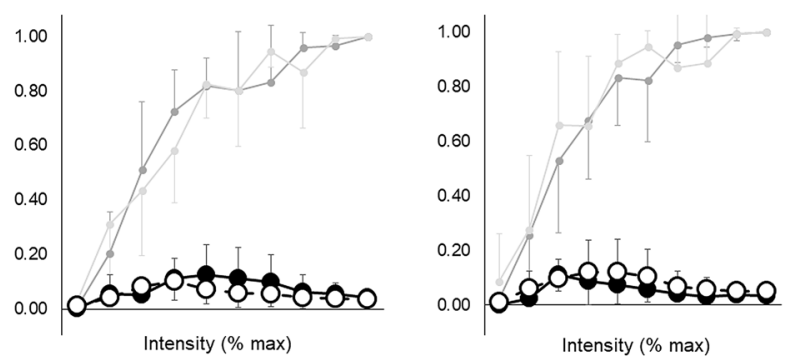

(portion 1: from 0 to $10 \%$ of maximal intensity; portion 2: from 11 to $20 \%$ of maximal intensity; ...; portion 10 : from 91 to $100 \%$ of maximal intensity). Open circles are responses recorded at PRE, and full circles are responses recorded at POST. Associated M-waves to each H-reflex, also normalized by the corresponding maximal M-wave, are represented in light gray for PRE and dark gray for POST. *Significant PRE-to-POST response at $p<0.05$ (dark: for $H / M_{\mathrm{MAX}}$ and gray for $\left.M_{\mathrm{atH}} / M_{\mathrm{MAX}}\right)$

colleagues in 2006 (Gondin et al. 2006) and Maffiuletti and colleagues in 2001 (Maffiuletti et al. 2001) following NMES training induced by maximal tolerable stimulation intensity, leading to considerable evoked force by subjects throughout each session (between 50 and $70 \%$ MVC for both studies) contrary to the present study. Therefore, it seems that NMES training is an effective stimulus for strength development even when the level of evoked force during training is relatively low.

In the present study, no architectural changes have been observed, suggesting that the short training period associated with low levels of evoked force does not induce a sufficient mechanical stress. Indeed, this adaptation has been 
Fig. 4 Plantar flexors V-wave and superimposed $\mathrm{H}$-reflex of the four groups. $V / M_{\mathrm{SUP}}$ and $H_{\text {SUP }} / M_{\text {SUP }}$ ratios are depicted for the four groups: the control group (CONTROL) and the three trained groups: neuromuscular electrical stimulation only (NMES), combined NMES and motor imagery (NMES + MI) and MI only (MI). Ratios before (PRE, white bars) and after (POST, black bars) are shown for the three tested muscles in $\mathbf{a}$ and $\mathbf{b}$ for soleus muscle, $\mathbf{c}$ and d for gastrocnemius medialis, and $\mathbf{e}$ and $\mathbf{f}$ for gastrocnemius lateralis. *** Significant PREPOST difference at $p<0.05$ and $p<0.01$, respectively
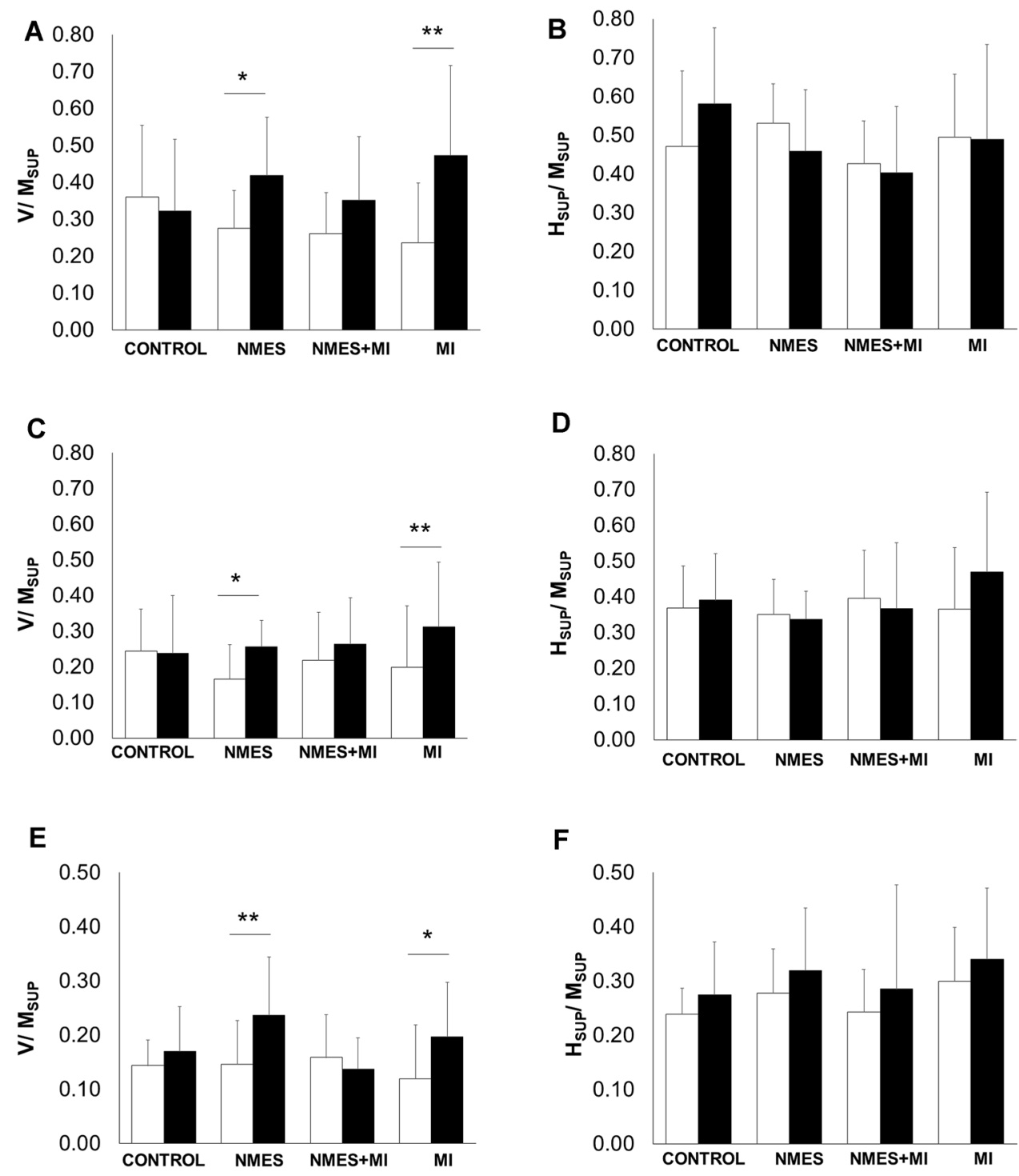

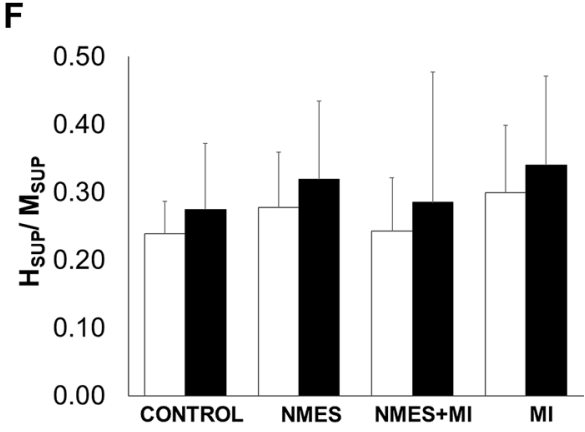

obtained after longer NMES training periods using higher stimulation intensities (Gondin et al. 2005; Lee and Yoon 2009). Nevertheless, some other changes at muscular levels were still observed.

Indeed, the peak twitch (Pt) and SOL $M_{\mathrm{MAX}}$ were increased from PRE to POST NMES training while GM $M_{\mathrm{MAX}}$ and GL $M_{\mathrm{MAX}}$ remained unchanged. An increase in Pt after NMES has already been shown in a few studies (Maffiuletti et al. 2003; Colson et al. 2009). Taken together, those changes led to a significant increase in electromechanical efficiency ratio (EME) found for NMES group only, indicating an improvement in excitation/contraction coupling efficiency. This parameter reflects the entire sequence of reactions from the spread of the action potential across the neuromuscular junction to the release of $\mathrm{Ca}^{2+}$ and the crossbridge interaction (Sandow 1952; Melzer et al. 1995). Thus, NMES training may have induced a more important release in calcium and/or a higher calcium sensitivity of contractile proteins. Through percutaneous muscle biopsies taken from the vastus lateralis muscle, a study of Russ and colleagues (2012) (Russ et al. 2012) showed that after NMES training (30\% MVC, $80 \mathrm{~Hz}$ for 5 weeks) the peak rate of $\mathrm{Ca}^{2+}$ release was significantly enhanced ( 16\%) (Russ et al. 2012). The lack of structural changes highlighted that peak twitch and EME evolution after NMES training rather originated from muscle intracellular processes.

It should be noticed that, in addition to an improvement in excitation/contraction coupling efficiency, higher nervous levels seemed involved in the strength gains observed after NMES training. NMES evokes action potentials in both intramuscular nerve branches (Hultman et al. 1983) and cutaneous receptors, thus also resulting in reflexive recruitment of spinal motoneurons (Collins et al. 2001). In the present study, since $H_{\text {SUP }} / M_{\text {SUP }}$ ratio of all three muscles was unaffected, spinal modulation seemed poorly involved in the strength gains associated with NMES. 
Although spinal excitability during MVC remained unchanged, it decreased at rest following NMES training. This decrease was accompanied by an increase in submaximal M-waves associated with $\mathrm{H}$-reflexes, i.e. $M_{\mathrm{atH}}$, in all muscles (Fig. 3). Such an enhancement of $M_{\mathrm{atH}}$ can induce an increase in antidromic current elicited by electrical impulses generated in motor axons by nerve stimulation (Piscione et al. 2012). This may have therefore canceled the reflexive activation, leading to lower H-reflexes at 40 and 50\% $M_{\mathrm{MAX}}$ intensity in POST tests. At these intensities, the positive correlation between such H-reflex depression and the increase in $M_{\mathrm{atH}} / M_{\mathrm{MAX}}$ supports this hypothesis. It emphasizes the fact that the so-observed decrease in $H / M_{\mathrm{MAX}}$ may not necessarily be due to a depression of spinal excitability per se. NMES leads to both direct and indirect activation of motor axons toward the muscle (Trimble and Enoka 1991). Here, the direct and repetitive activation during training may have caused an increase in axonal excitability leading, for submaximal nerve stimulation intensities, to a higher number of recruited motor units. Nevertheless, the measure of H-reflex does not necessarily reflect all the structures acting at the spinal level. An increase in spinal presynaptic inhibition following acute NMES training has been previously observed (Grosprêtre et al. 2018). Such an adaptation could also play a role in the decrease in rest H-reflex following NMES training.

However, NMES afferent or indirect activation led to plasticity of other nervous levels, as interpreted from the results of $V / M_{\mathrm{SUP}}$ and $H_{\mathrm{SUP}} / M_{\mathrm{SUP}}$. Measured during voluntary contraction, the $\mathrm{V}$-wave reflects motoneuron activation through afferents which benefited from the collision occurring between orthodromic motor action potentials elicited by the descending voluntary input to the motoneuron pool and the antidromic current generated in the motor axons by the external stimulation (Aagaard et al. 2002). Therefore, it is assumed that the magnitude of the collision is related to the descending motor command (Grosprêtre and Martin 2014). Nonetheless, by being a reflexive response, V-wave amplitude also involves motoneuron responsiveness (e.g., changes of intrinsic membrane properties and discharge rate), synaptic transmission efficacy at Ia afferent terminals (e.g., presynaptic inhibition), and/or postsynaptic inhibition (Carroll et al. 2011). These mechanisms are usually ruled out by measuring also the superimposed H-reflex to the same level of contraction, i.e., $H_{\text {Sup }}$. Consequently, it has been assumed that in the absence of any $H_{\text {SUP }}$ modulation, the increase of $\mathrm{V}$-wave amplitude reflects an increase of supraspinal activation, which enhances descending volitional drive (i.e., recruitment and/or motoneuron firing frequency) to the muscle (Aagaard et al. 2002), as it can be observed in the present NMES training. Such results have already been observed after 15 sessions of NMES applied over the triceps surae (Gondin et al. 2006), where the change in V-wave amplitude has been attributed to the cortical influence of NMES. To corroborate this hypothesis, previous works have already shown by functional neuroimaging that premotor and primary motor cortices can be activated during NMES (Han et al. 2003). It has been postulated that the brain activation induced by NMES, although not being systematically associated with changes at spinal level, might then imply a larger loop involving the sensory motor cortex and the motor cortex, both being interconnected. Such supraspinal action of NMES might then be one of the main mechanisms involved in such an increase in $\mathrm{V}$-wave.

\section{MI training alone}

In the present study, MI training group also increased their plantar flexor muscles MVC by $13.8 \pm 5.6 \%$. Although training protocols vary, strength gains of a higher magnitude have already been reported with MI modality (e.g., Yue and Cole 1992: $+22 \%$ after 4 weeks; Zijdewind et al. 2003: $+36 \%$ after 7 weeks and Grosprêtre et al. 2017: $+9.6 \%$ after 1 week). Contrary to NMES, this gain has been attributed solely to neural adaptations rather than muscular changes (Ranganathan et al. 2004). In the present study, the lack of changes in muscle architecture and any parameters related to the excitation/contraction coupling (M-waves, twitches) following MI training also argue for a pure nervous plasticity.

At first, cortical reorganization, supposedly leading to an optimization of the central command and/or a better coordination of muscle activation (Ranganathan et al. 2004) has been proposed as the main mechanism involved. The increase in $V / M_{\text {SUP }}$ ratio in SOL, GM and GL found in this study, without change in superimposed H-reflex $\left(H_{\text {SUP }}\right)$ after MI training also suggests a supraspinal adaptation. In line with preceding arguments regarding NMES-induced plasticity, such an increase in V-wave might occur because MI involves a particular activation of premotor and primary motor cortices (Decety et al. 1994). The increase of the motor evoked potential by transcranial magnetic stimulation observed in previous literature also argues for an increased excitability of the pathway from brain motor regions to the muscle (Grosprêtre et al. 2016). A previous work already showed that one week of daily MI practice led to an increase in triceps surae V-wave amplitude (Grosprêtre et al. 2017), emphasizing the benefits of MI to improve the ability to produce a great cortical descending command. However, in this last work such as in the present study, the measurement of rest spinal excitability PRE- and POST-MI training highlighted the involvement of another possible mechanism that implies spinal networks in the so-observed strength gains.

Noteworthy, significantly higher rest $H / M_{\mathrm{MAX}}$ ratios in both SOL and GM muscles were observed at $30 \%$ and $40 \%$ $M_{\mathrm{MAX}}$ intensity in POST tests, revealing an increase in rest spinal excitability after MI training. The lack of change in 
GL muscle could indicate that not all muscles have the same sensitivity to MI-induced plasticity. However, it should be pointed out that $H / M_{\mathrm{MAX}}$ ratios of GL muscles were low with a high variability, also explaining a lack of statistical changes with MI training despite low PRE-to-POST $p$ values ( $p=0.11$ ). Regarding SOL and GM, the increase in $H / M_{\mathrm{MAX}}$ ratio could not be the result of changes in stimulation conditions onto the posterior tibial nerve since no change in M-waves accompanying H-reflexes $\left(M_{\mathrm{atH}} / M_{\mathrm{MAX}}\right)$ was observed on both muscles (Grosprêtre and Martin 2012). Combined with the lack of change of superimposed H-reflex $\left(H_{\mathrm{SUP}} / M_{\mathrm{SUP}}\right)$, these higher $H / M_{\mathrm{MAX}}$ ratios indicated that MI did not involve a global arousal of spinal circuitry but has impacted specific circuits. Indeed, while $H_{\text {SUP }}$ involves both PRE- and POST-synaptic mechanisms, rest H-reflex changes are more likely attributed to presynaptic mechanisms at the level of Ia afferent-to-alpha motoneuron synapse (Zehr 2002; Misiaszek 2003) and to the excitability of the motoneuron itself (Grosprêtre et al. 2017). It is suggested that either the low-threshold motoneurons have become more excitable or the synapses of the Ia primary afferents became more efficient (Piscione et al. 2012). Indeed, MI was recently shown to result in a sub-threshold cortical motor output that could modulate the activity of spinal structures that mediates presynaptic inhibition of Ia terminal onto alpha motoneurons, i.e., the primary afferent depolarization interneurons (Grosprêtre et al. 2019). Overall, this put in evidence that such a partial activation of spinal circuitry during MI, when repeated in a training-designed protocol with multiple sessions of MI practice, might lead to a global increase in rest spinal excitability. Therefore, the neural adaptations accompanying the observed strength gains involved a larger part of the corticospinal system than usually expected, including both supraspinal and spinal plasticity.

\section{Combined NMES + MI training}

Surprisingly, the combination of MI and NMES in the present study did not lead to observe any increase in performance, and in any of the peripheral and central factors measured, contrary to either modality performed alone. This lack of effect of NMES + MI is possibly explained by two hypotheses.

First, to provide the same training volume (total number of contractions) it should be reminded that the number of contractions was reduced for both NMES and MI in this group, as compared to NMES and MI groups. Therefore, half of the NMES program and half of the MI program have been performed in the NMES + MI group. Both may then represent an insufficient stimulus, at muscle level for NMES and nervous level for MI, to provide significant gains. This should indicate that gains in NMES and in MI do not involve the same mechanisms and that performing half of each does not lead to $100 \%$ or more of the NMES or MI gains. A longer training program with this design might reveal strength gains, arguing in favor of a dose-response effect.

However, it can be noticed that despite twice the number of evoked contractions, the NMES group did not exhibit a doubled total TTI as compared to the NMES + MI group. This can be explained by the fact that the intensity used during NMES to match $20 \%$ MVC was set only on one contraction and was not readjusted during the session. Thus, this level of $20 \%$ MVC was not maintained during the whole NMES session. The decrease in force can be due on one hand to fatigue caused by the non-physiological (spatially fixed and synchronous) motor unit recruitment (Gregory and Bickel 2005; Bickel et al. 2011) as compared to voluntary contractions (Theurel et al. 2007) and on the other hand to a reduction in the number of motor units recruited due to changes in axonal excitability (Matkowski et al. 2015). Conversely, in the NMES + MI group, sets of 5 NMES contractions were alternated with sets of 5 imagined contractions. This recovery allowed NMES contractions to remain closer to $20 \%$ MVC along the session. But still, this muscular treatment was not a sufficient stimulus to induce significant neuromuscular plasticity and strength gains in the combined group as opposed to the NMES-training group. The lack of effect in the combined group could corroborate the dose-effect response of MI training programs (Paravlic et al. 2018), the duration and number of repetitions provided in this group being insufficient to provide the usually observed MI-induced plasticity.

The second hypothesis of this lack of effect in the NMES + MI group lies in a possible antagonist effect of NMES and MI, which possibly canceled the expected summation of NMES and MI effects. As mentioned above, the afferent feedback induced by NMES led to a modulation of a larger loop involving a cortical effect. This supraspinal influence of NMES might also compete with the central activation by MI, since it could be qualitatively different. Indeed, NMES brain activation lies in the relationship between sensory and motor regions, while MI reflects activation directly initiated in the cortex. While several cortical inhibitory mechanisms were proposed during movement preparation of imagined action to prevent the movement from happening (Lebon et al. 2019), this might reveal as contradictory influence between the sensory and motor information provided by NMES. At spinal level, the concurrent effect of MI and NMES is even more apparent, as illustrated by the opposite effects of NMES and MI observed on H-reflex recruitment curves. If $\mathrm{MI}$ and NMES might involve the same spinal circuitry, i.e., the presynaptic inhibitory processes, the direction of the modulation is opposite between these two forms of treatment of the spinal cord. While NMES was shown to induce acute increase in spinal presynaptic inhibition (Grosprêtre et al. 2018), in contrast the effect of 
an acute MI session led to the removal of presynaptic inhibition (Grosprêtre et al. 2019). Therefore, when implemented in the same training session, both modalities might compete, canceling the effect that one should have when performed alone. The acute effect of this combination should be assessed in further research.

Another combination design simultaneously combining MI and NMES, i.e. imagining the contraction while it is electrically evoked, was acutely tested by Kaneko and colleagues (Kaneko et al. 2014). They showed that corticospinal tract excitability was acutely increased when NMES and MI were used simultaneously. MEP amplitude reached a level similar to that measured during brief voluntary muscle contraction, even though voluntary muscle contractions were absent during the combined condition. This could then be an alternative to combine efficiently NMES and MI.

\section{Practical recommendations}

Improving plantar flexors neuromuscular function without involving an important training load is of importance, particularly for frail populations. This muscle group contributes to many functional tasks, more particularly related to balance in upright standing posture. In that matter, plantar flexors MVC appeared to be a critical factor for falling in the elderly (Cattagni et al. 2014).

If reaching functional arousal such as increasing maximal force, the present study raised that MI and NMES can lead to similar gains. Therefore, the choice of one or the other modality could depend upon the strength and weakness of each method. While being very effective to induce neuromuscular plasticity, NMES needs specific material and is subjected to a particular expertise of the operator (electrodes positioning, setting of the device) and more importantly, present the inherent drawback of being uncomfortable for many participants, even when not using a maximal tolerable intensity. On the opposite, MI represents a costless, safe and simple method to induce strength gains. But one of the main drawbacks of MI lies in the expertise of the participant itself to mentally represent an action, and in its commitment to perform the task. Besides being easily boring, which can lead to a decrease in the participant's dedication to perform the task due to motivational aspects, MI represents a high cognitive process that requires learning and might be hardly manageable for populations with mental disabilities. Therefore, both MI and NMES have their specificities and might be chosen according to the context and the targeted population. But the present study raised that MI and NMES involve, above all, different mechanisms in the training induced plasticity. This should also be considered when choosing to implement one or the other modality for training purpose. While NMES seems to be a very effective stimulus to provide changes at the level of the neuromuscular junction, even in absence of muscle structural changes, MI mostly provides central plasticity.

While combining MI with other sensorial inputs has been widely recommended to magnify the effect of such training modality, the present study raised that antagonist effect might also interfere with the gains in motor performance that should be emphasized by such a modality of training.

\section{Conclusion and perspectives}

This study showed similar gains in MVC between NMES and MI trainings, both relying on neural adaptations which, overall, involved a larger part of the corticospinal system than usually expected, including both supraspinal and spinal plasticity.

Unexpectedly, alternating NMES and MI in the same training session did not imply strength gains, possibly explained by different hypotheses, such as (1) the insufficient stimulus provided at the peripheral level for NMES and at central level for MI or (2) concurrent effects between both modalities.

It is therefore suggested that the present MI and NMES protocols performed alone are more efficient than alternating half of both in a similar training design. Yet, another training design simultaneously combining MI and NMES, i.e. imagining the contraction while it is electrically evoked, might be an alternative. Nonetheless, the acute or chronic effects of repeating this modality of NMES and MI combination have not been investigated and could be the subject of future research.

Author contributions AM and SG: conceived and designed research; $\mathrm{AB}$ : conducted experiments and extracted results; SG: ran statistical analyses and designed graphs and tables; AB, AM and SG: analyzed data; AB: wrote the manuscript. All authors read and approved the manuscript.

Funding No funding was received for this work.

Data availability The results of the study are presented clearly, honestly, and without fabrication, falsification, or inappropriate data manipulation. All data are available if needed.

\section{Compliance with ethical standards}

Conflict of interest There is no conflict of interest or competing interests.

Ethical approval The experimental design was approved by the regional ethic committee (CPP COOM III number 2017-A00064-49; Clinical trial.gov identifier NCT03334526) and conducted in conformity with the latest version of the Declaration of Helsinki. 
Informed consent Participants gave written informed consent to participate in the present study and to its publication.

Open Access This article is licensed under a Creative Commons Attribution 4.0 International License, which permits use, sharing, adaptation, distribution and reproduction in any medium or format, as long as you give appropriate credit to the original author(s) and the source, provide a link to the Creative Commons licence, and indicate if changes were made. The images or other third party material in this article are included in the article's Creative Commons licence, unless indicated otherwise in a credit line to the material. If material is not included in the article's Creative Commons licence and your intended use is not permitted by statutory regulation or exceeds the permitted use, you will need to obtain permission directly from the copyright holder. To view a copy of this licence, visit http://creativecommons.org/licenses/by/4.0/.

\section{References}

Aagaard P (2003) Training-induced changes in neural function. Exerc Sport Sci Rev 31:61-67. https://doi.org/10.1097/00003677-20030 4000-00002

Aagaard P, Simonsen EB, Andersen JL et al (2002) Neural adaptation to resistance training: changes in evoked V-wave and H-reflex responses. J Appl Physiol 92:2309-2318. https://doi.org/10.1152/ japplphysiol.01185.2001

Bickel CS, Gregory CM, Dean JC (2011) Motor unit recruitment during neuromuscular electrical stimulation: a critical appraisal. Eur J Appl Physiol 111:2399. https://doi.org/10.1007/s0042 1-011-2128-4

Carroll TJ, Selvanayagam VS, Riek S, Semmler JG (2011) Neural adaptations to strength training: moving beyond transcranial magnetic stimulation and reflex studies. Acta Physiol 202:119-140. https://doi.org/10.1111/j.1748-1716.2011.02271.x

Cattagni T, Scaglioni G, Laroche D et al (2014) Ankle muscle strength discriminates fallers from non-fallers. Front Aging Neurosci. https ://doi.org/10.3389/fnagi.2014.00336

Collins DF, Burke D, Gandevia SC (2001) Large involuntary forces consistent with plateau-like behavior of human motoneurons. J Neurosci 21:4059-4065. https://doi.org/10.1523/jneurosci.21-1104059.2001

Colson SS, Martin A, Van Hoecke J (2009) Effects of electromyostimulation versus voluntary isometric training on elbow flexor muscle strength. J Electromyogr Kinesiol 19:311-319. https://doi. org/10.1016/j.jelekin.2008.05.009

Decety J, Perani D, Jeannerod M et al (1994) Mapping motor representations with positron emission tomography. Nature 371:600-602. https://doi.org/10.1038/371600a0

Gondin J, Guette M, Ballay Y, Martin A (2005) Electromyostimulation training effects on neural drive and muscle architecture. Med Sci Sports Exerc 37:1291-1299. https://doi.org/10.1249/01. mss.0000175090.49048.41

Gondin J, Duclay J, Martin A (2006) Soleus- and gastrocnemiievoked V-wave responses increase after neuromuscular electrical stimulation training. J Neurophysiol 95:3328-3335. https://doi. org/10.1152/jn.01002.2005

Gregory CM, Bickel CS (2005) Recruitment patterns in human skeletal muscle during electrical stimulation. Phys Ther 85:358-364. https ://doi.org/10.1093/ptj/85.4.358

Grosprêtre S, Martin A (2012) H reflex and spinal excitability: methodological considerations. J Neurophysiol 107:1649-1654. https ://doi.org/10.1152/jn.00611.2011
Grosprêtre S, Martin A (2014) Conditioning effect of transcranial magnetic stimulation evoking motor-evoked potential on v-wave response. Physiol Rep 2:e12191. https://doi.org/10.14814/ phy2.12191

Grosprêtre S, Ruffino C, Lebon F (2016) Motor imagery and corticospinal excitability: a review. Eur J Sport Sci 16:317-324. https:// doi.org/10.1080/17461391.2015.1024756

Grosprêtre S, Jacquet T, Lebon F et al (2017) Neural mechanisms of strength increase after 1-week motor imagery training. Eur J Sport Sci 18:209-218. https://doi.org/10.1080/17461391.2017.1415377

Grosprêtre S, Gueugneau N, Martin A, Lepers R (2018) Presynaptic inhibition mechanisms may subserve the spinal excitability modulation induced by neuromuscular electrical stimulation. J Electromyogr Kinesiol 40:95-101. https://doi.org/10.1016/j.jelek in.2018.04.012

Grosprêtre S, Lebon F, Papaxanthis C, Martin A (2019) Spinal plasticity with motor imagery practice. J Physiol 597:921-934. https:// doi.org/10.1113/JP276694

Guillot A, Collet C (2008) Construction of the motor imagery integrative model in sport: a review and theoretical investigation of motor imagery use. Int Rev Sport Exerc Physiol 1:31-45. https://doi. org/10.1080/17509840701823139

Hainaut K, Duchateau J (1992) Neuromuscular electrical stimulation and voluntary exercise. Sport Med 14:100-113. https://doi. org/10.2165/00007256-199214020-00003

Hall CR, Martin KA (1997) Measuring movement imagery abilities: a revision of the Movement Imagery Questionnaire. J Ment Imag 21:143-154

Han BS, Jang SH, Chang Y et al (2003) Functional magnetic resonance image finding of cortical activation by neuromuscular electrical stimulation on wrist extensor muscles. Am J Phys Med Rehabil 82:17-20. https://doi.org/10.1097/00002060-200301000-00003

Hultman E, Sjöholm H, Jäderholm-Ek I, Krynicki J (1983) Evaluation of methods for electrical stimulation of human skeletal muscle in situ. Pflügers Arch Eur J Physiol 398:139-141. https://doi. org/10.1007/BF00581062

Jeannerod M (1994) The representing brain: neural correlates of motor intention and imagery. Behav Brain Sci. https://doi.org/10.1017/ S0140525X00034026

Kaneko F, Hayami T, Aoyama T, Kizuka T (2014) Motor imagery and electrical stimulation reproduce corticospinal excitability at levels similar to voluntary muscle contraction. J Neuroeng Rehabil 11:94. https://doi.org/10.1186/1743-0003-11-94

Kidgell DJ, Bonanno DR, Frazer AK et al (2017) Corticospinal responses following strength training: a systematic review and meta-analysis. Eur J Neurosci 46:2648-2661. https://doi. org/10.1111/ejn.13710

Laughman RK, Youdas JW, Garrett TR, Chao EYS (1983) Strength changes in the normal quadriceps femoris muscle as a result of electrical stimulation. Phys Ther 63:494-499. https://doi. org/10.1093/ptj/63.4.494

Lebon F, Ruffino C, Greenhouse I et al (2019) The neural specificity of movement preparation during actual and imagined movements. Cereb Cortex 29:689-700. https://doi.org/10.1093/cercor/bhx350

Lee J-W, Yoon S-W (2009) The effects of neuromuscular electrical stimulation on skeletal muscle architecture and qualitative properties in vivo. Int J Contents 5:35-39

Maffiuletti NA (2010) Physiological and methodological considerations for the use of neuromuscular electrical stimulation. Eur J Appl Physiol 110:223-234. https://doi.org/10.1007/s00421-010-1502-y

Maffiuletti NA, Pensini M, Martin A (2001) Activation of human plantar flexor muscles increases after electromyostimulation training. J Appl Physiol 92:1383-1392. https://doi.org/10.1152/japplphysi ol.00884.2001 
Maffiuletti NA, Dugnani S, Folz M et al (2002) Effect of combined electrostimulation and plyometric training on vertical jump height. Med Sci Sport Exerc 34:1638-1644

Maffiuletti NA, Pensini M, Scaglioni G et al (2003) Effect of electromyostimulation training on soleus and gastrocnemii $\mathrm{H}-$ and T-reflex properties. Eur J Appl Physiol 90:601-607. https://doi. org/10.1007/s00421-003-0914-3

Matkowski B, Lepers R, Martin A (2015) Torque decrease during submaximal evoked contractions of the quadriceps muscle is linked not only to muscle fatigue. J Appl Physiol 118:1136-1144. https ://doi.org/10.1152/japplphysiol.00553.2014

Melzer W, Herrmann-Frank A, Hc L (1995) The role of $\mathrm{Ca}^{2+}$ ions in excitation-contraction coupling of skeletal muscle fibers. Biochim Biophys Acta 1241:59-116

Misiaszek JE (2003) The H-reflex as a tool in neurophysiology: its limitations and uses in understanding nervous system function. Muscle Nerve 28:144-160. https://doi.org/10.1002/mus.10372

Paravlic AH, Slimani M, Tod D et al (2018) Effects and dose-response relationships of motor imagery practice on strength development in healthy adult populations: a systematic review and meta-analysis. Sport Med 48:1165-1187. https://doi.org/10.1007/s4027 9-018-0874-8

Piscione J, Grosset JF, Gamet D, Pérot C (2012) Are H-reflex and M-wave recruitment curve parameters related to aerobic capacity? Appl Physiol Nutr Metab 37:990-996. https://doi.org/10.1139/ H2012-078

Ranganathan VK, Siemionow V, Liu JZ et al (2004) From mental power to muscle power-gaining strength by using the mind. Neuropsychologia 42:944-956. https://doi.org/10.1016/j.neuro psychologia.2003.11.018

Russ DW, Clark BC, Krause J, Hagerman FC (2012) Development of a neuromuscular electrical stimulation protocol for sprint training. Med Sci Sports Exerc 44:1810-1819. https://doi.org/10.1249/ MSS.0b013e31825423f1

Sandow A (1952) Excitation-contraction coupling in muscular response. Yale J Biol Med 25:176-201

Schieppati M (1987) The Hoffmann reflex: a means of assessing spinal reflex excitability and its descending control in man. Prog Neurobiol 28:345-376. https://doi.org/10.1016/0301-0082(87)90007-4
Seyri KM, Maffiuletti NA (2011) Effect of electromyostimulation training on muscle strength and sports performance. Strength Cond J 33:70-75

Sidaway B, Trzaska AR (2005) Can mental practice increase ankle Dorsiflexor torque? Phys Ther 85:1053-1060

Stevens JE, Mizner RL, Snyder-Mackler L (2004) Neuromuscular electrical stimulation for quadriceps muscle strengthening after bilateral total knee arthroplasty: a case series. J Orthop Sports Phys Ther 34:21-29. https://doi.org/10.2519/jospt.2004.34.1.21

Tallent J, Goodall S, Gibbon KC et al (2017) Enhanced corticospinal excitability and volitional drive in response to shortening and lengthening strength training and changes following detraining. Front Physiol 8:57. https://doi.org/10.3389/fphys.2017.00057

Theurel J, Lepers R, Pardon L, Maffiuletti NA (2007) Differences in cardiorespiratory and neuromuscular responses between voluntary and stimulated contractions of the quadriceps femoris muscle. Respir Physiol Neurobiol 157:341-347. https://doi.org/10.1016/j. resp.2006.12.002

Trimble MH, Enoka RM (1991) Mechanisms underlying the training effects associated with neuromuscular electrical stimulation. Phys Ther 71:273-280. https://doi.org/10.1093/ptj/71.4.273

Yue G, Cole KJ (1992) Strength increases from the motor program: comparison of training with maximal voluntary and imagined muscle contractions. J Neurophysiol 167:1114-1123. https://doi. org/10.1152/jn.1992.67.5.1114

Zehr EP (2002) Considerations for use of the Hoffmann reflex in exercise studies. Eur J Appl Physiol 86:455-468. https://doi. org/10.1007/s00421-002-0577-5

Zijdewind I, Toering ST, Bessem B et al (2003) Effects of imagery motor training on torque production of ankle plantar flexor muscles. Muscle Nerve 28:168-173

Publisher's Note Springer Nature remains neutral with regard to jurisdictional claims in published maps and institutional affiliations. 